\title{
TERT promoter mutations: a genetic signature of benign and malignant thyroid tumours occurring in the context of tinea capitis irradiation
}

\author{
Paula Boaventura 1,2, Rui Batista1,2, Ana Pestana1,2, Marta Reis ${ }^{1,2}$, Adélia Mendes ${ }^{1,2}$, \\ Catarina Eloy ${ }^{1,2}$, Manuel Sobrinho-Simões ${ }^{1,2,3,4}$ and Paula Soares ${ }^{1,2,3}$ \\ ${ }^{1}$ IPATIMUP - Institute of Molecular Pathology and Immunology of the University of Porto, Porto, \\ Portugal, 2i3S - Instituto de Investigação e Inovação em Saúde, Universidade do Porto, Porto, Portugal, \\ ${ }^{3}$ Faculty of Medicine, University of Porto, Porto, Portugal, and ${ }^{4}$ Department of Pathology, \\ Centro Hospitalar de S. João, Porto, Portugal
}

\author{
Correspondence \\ should be addressed \\ to P Boaventura \\ Email \\ mboaventura@ipatimup.pt
}

\begin{abstract}
Objective: The aim of this study is to evaluate the frequency and molecular characteristics of TERTp mutations in thyroid adenomas and carcinomas occurring in the low-dose radiation exposure tinea capitis setting. Design and methods: Twenty-seven patients with 34 well-differentiated thyroid carcinomas and 28 patients with 29 follicular adenomas diagnosed in a Portuguese tinea capitis cohort were studied. Blood samples were obtained from all the patients. Screening for TERTp mutations was performed by PCR amplification followed by Sanger sequencing. A series of 33 sporadic thyroid adenomas was used as control.

Results: TERTp mutations were detected in six of the 28 patients with adenoma (21.4\%) and in four of the 27 patients with carcinoma (14.8\%). Three tumours (two carcinomas and one adenoma) had the tandem mutation $-124 /-125$ GG>AA (30.0\%), whereas the remaining seven had the $-124 \mathrm{G}>\mathrm{A}$. The $20.7 \%$ frequency of TERTp mutations in adenomas contrasts with the absence of mutations in the adenomas from the control group and from most series on record, whereas the one found in carcinomas $(11.8 \%)$ is similar to those reported in the literature for sporadic carcinomas. Conclusion: TERTp mutations, including the tandem mutation -124/-125 GG>AA not described previously in thyroid tumours, appear to represent a genetic signature for thyroid tumours in patients submitted to low-dose X-ray irradiation. The high frequency of TERTp mutations in the adenomas of our cohort contrasts with their absence in sporadically occurring, as well as in adenomas of the Chernobyl series.
\end{abstract}

\section{Introduction}

Tinea capitis is a fungal disease of the scalp that attained epidemic proportions in the 5th-6th decades of last century due to the lack of anti-fungal oral treatment (1). Before the appearance of griseofulvin, the best way to eradicate the disease was to induce scalp epilation with $\mathrm{X}$-ray irradiation using the Adamson-Kienbock technique $(2,3)$. The children received $7 \mathrm{~Gy}$ in the scalp (4), $1.5 \mathrm{~Gy}$ in the brain (5) and 0.045-0.5 Gy in the thyroid (6).
It is well recognised that X-ray radiation exposure is associated with cancer development (7). Increased frequencies of basal cell carcinoma $(8,9)$, thyroid carcinoma $(1,6)$ and meningioma (5) have been shown in the tinea capitis cohorts.

We have been following such a cohort since 2006 encompassing individuals who were submitted to scalp $\mathrm{X}$-ray epilation in Portugal at the former Dispensário
() 2017 European Society of Endocrinology Printed in Great Britain
Published by Bioscientifica Ltd. 
Central de Higiene Social do Porto (DCHSP) between 1950 and $1963(1,10)$. In thyroid carcinomas from these patients (with a long latency period), we found that the genetic alterations profile was more similar to that of the sporadic context, regarding for example, a higher frequency of $B R A F$ mutation than that of the Chernobyl radiation-induced context $(11,12)$. A study from our group had previously shown that BRAF mutations were not a major event in early onset post-Chernobyl childhood thyroid carcinomas (13).

Regarding the presence of RET/PTC rearrangements in radiation context, conflicting results have been presented regarding radiation exposure, age at exposure and histology of the tumours $(14,15,16)$. Elisei et al. stated that the presence of RET/PTC rearrangements is not higher in radiation-induced thyroid tumours than in sporadic, is not different after exposure to radioiodine or external radiation and is not associated with the young age of the patients also (17).

In Chernobyl studies, it was shown that the frequency of BRAF increases (18), whereas the frequency of RET/PTC rearrangements decreases with an increased latency period between exposure and clinical presentation $(7,19,20)$. These data fit with the hypothesis advanced by Williams et al. that Chernobyl-related carcinomas will appear in 'waves', a first 'wave' with very short latency, in very young patients and showing a distinct genetic profile (frequent RET/PTC rearrangements and low BRAF mutations), and a late-onset 'wave' affecting older patients (20-40 years) and displaying a genetic profile similar to that found in adult sporadic thyroid carcinoma (low RET/PTC rearrangements and high BRAF mutation) $(19,20)$. In fact, Dinets et al. (21) found frequent $B R A F$ mutations in a series of 70 adult patients exposed in childhood or as teenagers to the Chernobyl radioactive fallout and surgically treated between 2004 and 2008 (late-onset lesions) (21). In summary, these observations suggest that molecular changes in thyroid cancer after the Chernobyl accident mirror those occurring spontaneously, age (or time) of onset being more relevant than the etiopathogenic factor.

Telomerase, a ribonucleoprotein that maintains telomere length at the end of the chromosomes, is expressed at functionally significant levels in several human cancer cells, promoting their immortality (22). The TERT gene encodes the reverse transcriptase component of telomerase. Mutations in its promoter have been recently described in several cancers (23) and are particularly important as they provide the first evidence of driver mutations in the so-called dark matter of the human genome (24). They have been described in thyroid carcinomas where they are an indicator of clinically aggressive tumours $(25,26,27)$. There are limited data on the prevalence of TERTp mutations in radiationinduced thyroid carcinomas. To explore the influence of the external radiation context (X-rays), we decided to search for such mutations in thyroid tumours from the tinea capitis-irradiated cohort. As far as we know, TERT promoter mutations have never been studied in cancers associated with X-ray external radiation, namely in the tinea capitis cohort submitted to low-dose radiation in the thyroid.

\section{Subjects and methods}

\section{Patients}

We have previously observed from the clinical standpoint 1375 individuals from an original cohort of 5356 individuals who had undergone scalp irradiation for tinea capitis treatment during their childhood. Thirty-eight of those 1375 individuals were found to present thyroid carcinoma (2.8\%) (1). The molecular study was conducted using the DNA from the tinea capitis patients from whom we had retrieved thyroid carcinomas and/or adenomas, whenever material was available (11). We also used DNA from blood collected from all the patients to search for mutations in blood cells.

In total, we studied 34 carcinomas from 27 patients (five patients had two carcinomas and one patient had three carcinomas), and 29 adenomas from 28 patients, as one patient had two adenomas. Three patients presenting carcinomas and adenomas were included in both groups two with one adenoma and one carcinoma, and the third with two adenomas and two carcinomas. Thirty-two carcinomas were classified as papillary thyroid carcinoma (PTC): $40.6 \%$ classical variant and $59.4 \%$ follicular variant. The remaining two carcinomas were a follicular carcinoma (FTC) and a well-differentiated carcinoma no otherwise specified (WDT-NOS).

Additionally, we have studied a series of sporadically occurring follicular adenomas, retrieved from the files of Centro Hospitalar de S. João, comprising 33 adenomas from 33 non-irradiated individuals.

All the procedures were performed under strict ethical and confidentiality procedures according to the Portuguese ethical rules. The study has been approved by the Ethics Committee of the Hospital Pedro Hispano, and all the patients signed up an informed consent. 
Table 1 Clinicopathological characteristics and TERTp mutation frequency in thyroid carcinomas and thyroid adenomas of X-ray-irradiated patients.

\begin{tabular}{l} 
Clinicopathological characteristics \\
\hline Number of patients \\
Age at diagnosis (mean \pm S.D.) \\
Latency (mean \pm s.D.) \\
Age at irradiation (mean \pm S.D.) \\
Gender $(n(\%))$ \\
Female \\
Male \\
Irradiation dose $(n(\%)) *$ \\
325-400 R \\
$\quad \geq 630 \mathrm{R}$
\end{tabular}

\begin{tabular}{|c|c|c|}
\hline \multicolumn{3}{|c|}{ Thyroid carcinoma } \\
\hline Total & TERTp wt & TERTp mut \\
\hline 27 & 23 & 4 \\
\hline $56.7 \pm 6.7$ & $55.7 \pm 6.0$ & $62.0 \pm 8.7$ \\
\hline $50.2 \pm 6.3$ & $49.4 \pm 6.2$ & $54.5 \pm 5.7$ \\
\hline $6.4 \pm 2.9$ & $6.2 \pm 2.7$ & $7.3 \pm 4.3$ \\
\hline $19(70.4)$ & $16(69.6)$ & $3(75.0)$ \\
\hline $8(29.6)$ & $7(30.4)$ & $1(25.0)$ \\
\hline $24(92.3)$ & $20(90.9)$ & $4(100.0)$ \\
\hline $2(7.7)$ & $2(9.1)$ & $0(0.0)$ \\
\hline
\end{tabular}

\begin{tabular}{|c|c|c|}
\hline \multicolumn{3}{|c|}{ Thyroid adenoma } \\
\hline Total & TERTp wt & TERTp mut \\
\hline 28 & 22 & 6 \\
\hline $54.3 \pm 7.7$ & $54.6 \pm 7.6$ & $54.6 \pm 10.2$ \\
\hline $47.9 \pm 7.2$ & $48.0 \pm 6.8$ & $48.0 \pm 10.9$ \\
\hline $6.5 \pm 2.4$ & $6.7 \pm 2.4$ & $6.6 \pm 2.7$ \\
\hline $22(78.6)$ & $19(86.4)$ & $4(66.7)$ \\
\hline $6(21.4)$ & $3(13.6)$ & $2(33.3)$ \\
\hline $28(100.0)$ & $22(100.0)$ & $6(100.0)$ \\
\hline $0(0)$ & $0(0)$ & $0(0)$ \\
\hline
\end{tabular}

*One case of carcinoma without information on the dose received.

\section{Identification of TERTp mutations}

To screen for TERT promoter mutations, we analysed the hot spots previously identified by PCR followed by Sanger sequencing. TERT promoter mutation analysis was performed with the pair of primers forward TERTF, CAGCGCTGCCTGAAACTC; and reverse TERTR, GTCCTGCCCCTTCACCTT. Amplification of genomic DNA (25-100 ng) was performed by PCR using the QIAGEN multiplex PCR kit, using the following cycling conditions: $30 \mathrm{~s}$ at $95^{\circ} \mathrm{C}, 90 \mathrm{~s}$ at $62^{\circ} \mathrm{C}$ and $20 \mathrm{~s}$ at $72^{\circ} \mathrm{C}$ for 35 cycles. All PCR products were purified and directly sequenced on an ABI Prism $3130 x l$ Automatic sequencer (Perkin-Elmer) using the ABI Prism Dye Terminator Cycle sequencing kit (Perkin-Elmer). The sequencing reaction was performed in the forward direction, and an independent PCR amplification, both in the forward and reverse directions, was performed in the samples that were suspected to carry the mutations.

\section{Statistical analysis}

The PASW Statistics 23 program was used. Proportions were compared using $\chi^{2}$ test; the significance of differences between means was assessed by Student's unpaired $t$-test. A $P$ value $<0.05$ was considered statistically significant with a $95 \%$ confidence interval.

\section{Results}

In the non-irradiated setting, composed by 33 patients with thyroid adenomas, 27 women (81.8\%) and 6 men (18.2\%), with a mean age at diagnosis of $53.8 \pm 7.8$, no mutations were detected in TERT promoter. In the radiation-exposed setting, TERTp mutations were detected in four patients with thyroid carcinoma (14.8\%) and in six patients with thyroid adenoma (21.4\%). No mutations were found in the corresponding blood samples. The cases had been previously analysed for BRAF and NRAS mutations, RET/ PTC and PAX8PPARG rearrangements (11); only one $B R A F$-mutated carcinoma had TERTp mutation (Table 1). We detected a case with NRAS mutation, which was wild type for TERTp mutation.

Table 1 summarises the clinicopathological characteristics of the irradiated patients according to the mutation status. One patient presented one mutated adenoma, one wild-type adenoma and one wild-type carcinoma and another patient presented one mutated adenoma and two wild-type carcinomas (Table 2). The other eight patients with mutated tumours had only one tumour. In the carcinoma group, age at diagnosis (TERTpmut $62.0 \pm 8.7$ vs TERTpwt $55.7 \pm 6.0$ ), age at irradiation (TERTpmut $7.3 \pm 4.3$ vs TERTpwt $6.2 \pm 2.7$ ), and latency, defined as time between irradiation and

Table 2 Type of TERTp mutations found in the mutated tumours.

\begin{tabular}{lccc}
\hline Case & Adenomas & & Carcinomas \\
\cline { 1 - 2 } $2^{\mathrm{a}}$ & $-124: G>A$ & $W \mathrm{Wt}$ \\
$4^{\mathrm{b}}$ & $-124: G>A$ & Wt/wt \\
5 & - & $-124: G>A^{*}$ \\
7 & $-124 /-125: G G>A A$ & $-124: G>A^{*}$ \\
16 & - & - \\
17 & $-124: G>A$ & $-124 /-125: G G>A A^{*}$ \\
20 & $-124: G>A$ & - \\
23 & - & - \\
41 & $-124: G>A$ & $-124 /-125: G G>A A * *$ \\
45 & & - \\
\hline
\end{tabular}

apatient with two independent lesions; ${ }^{b}$ patient with three independent lesions; *papillary thyroid carcinoma; **well-differentiated thyroid carcinoma not otherwise specified. 
Table 3 TERTp mutation frequency according to the histological type and $B R A F$ mutation in the 34 carcinomas.

\begin{tabular}{l}
\hline \\
Number of carcinomas \\
Histological type \\
PTC \\
FTC \\
WDT-NOS \\
BRAF \\
BRAF wt \\
BRAF mut
\end{tabular}

\begin{tabular}{|c|c|c|}
\hline Total & TERTp wt & TERTp mut \\
\hline 34 & 30 & 4 \\
\hline 32 (94.1) & 29 & $3(75.0)$ \\
\hline $1(2.9)$ & 1 & $0(0)$ \\
\hline $1(2.9)$ & 0 & $1(25.0)$ \\
\hline $25(73.5)$ & 22 (73.3) & $3(75.0)$ \\
\hline $9(26.5)$ & $8(26.7)$ & $1(25.0)$ * \\
\hline
\end{tabular}

FTC, follicular thyroid carcinoma; PTC, papillary thyroid carcinoma; WDT-NOS, well-differentiated thyroid carcinoma not otherwise specified. *PTC.

surgery (TERTpmut $54.5 \pm 5.7$ vs TERTpwt $49.4 \pm 6.2$ ), were higher in TERT patients with mutated tumours, although the differences were not statistically significant. Regarding the adenoma group, age at diagnosis (TERTp

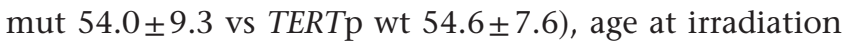
(TERTp mut $6.3 \pm 2.5$ vs TERTp wt $6.7 \pm 2.4$ ), and latency (TERTp mut $47.7 \pm 9.8$ vs TERTp wt $48.0 \pm 6.8$ ), were similar in patients with TERTp-mutated and -nonmutated tumours. No significant differences were found, in both groups, for gender and irradiation dose. Also, comparing together all the mutated lesions with the wildtype lesions did not reveal any significant differences in the aforementioned clinicopathological characteristics (data not shown). Regarding the size of the tumours, there was also no significant correlation with TERTp mutations, either considering all the mutated lesions (TERTp mut $1.6 \pm 1.2 \mathrm{~cm}$ vs TERTp wt $1.7 \pm 1.8 \mathrm{~cm}$ ) or comparing separately adenomas (TERTp mut $1.5 \pm 1.0 \mathrm{~cm}$ vs TERTp wt $2.7 \pm 2.3 \mathrm{~cm}$ ) and carcinomas (TERTp mut $1.8 \pm 1.5 \mathrm{~cm}$ vs TERTp wt $0.9 \pm 0.6 \mathrm{~cm})$.

The mutations comprised the $-124 \mathrm{G}>\mathrm{A}$ mutation that was present in six cases (five FTA and one PTC), and the tandem GG > AA mutation at position -124/-125 bp in three cases (one FTA, one PTC and one WDC-NOS); the tandem mutation was observed in $50 \%$ of the mutated thyroid carcinomas $(2 / 4)$ and in $16.7 \%$ of the mutated adenomas (1/6) (Table 2).

The histological subtype and the $B R A F^{V 600 E}$ mutation status of the carcinomas are presented in Table 3 . One of the TERTp-mutated tumours was a classic PTC, two were follicular variant of PTC and the fourth was a WDCNOS. There were no significant differences between wild-type and TERTp-mutated carcinomas concerning the PTC histological subtype or the $B R A F^{V 600 E}$ mutation status, but the series is too small to allow a meaningful comparison.

We observed TERTp mutations in 6 of the 29 adenomas $(20.7 \%)$ at variance with none in the 33 sporadically occurring adenomas and in the cases we have previously observed in other selected series $(25,28)$. TERTp mutations were detected in 3 of 32 PTCs $(9.2 \%)$. This percentage fits with those we had previously observed in PTC $(7.5 \%$ and $7.7 \%$ respectively) $(25,28)$. The same holds true regarding the group of well-differentiated carcinomas (encompassing PTC, FTC and WDC-NOS) (11.8\% in the present study, and $9.2 \%$ and $9.4 \%$ respectively) $(25,28)$. These data are summarised in Table 4.

\section{Discussion}

To better clarify the genetic profile of low-dose external radiation-induced thyroid tumours, we decided to search for TERTp mutations in thyroid tumours from the Portuguese tinea capitis cohort. For comparison, we analysed a similar series of non-radiation-exposed follicular adenomas, and also used, for comparative purposes, the results we have previously obtained in sporadically occurring thyroid tumours $(25,28)$.

In the radiation-exposed setting, we detected TERTp mutations in $14.8 \%$ of the patients with carcinoma (11.8\% of the carcinoma lesions) and in $21.4 \%$ of the patients with adenoma (20.7\% of the adenoma lesions). Regarding the carcinoma, the frequencies we detected in the present study are in the range of those found

Table 4 TERTp mutations in thyroid tumours series studied by our group.

\begin{tabular}{|c|c|c|c|c|}
\hline \multirow[b]{2}{*}{ Thyroid tumours } & \multicolumn{4}{|c|}{ TERTp mutations (n/total (\%)) } \\
\hline & $\begin{array}{l}\text { Present series } \\
\text { (X-irradiated) }\end{array}$ & $\begin{array}{l}\text { Present series (non- } \\
\text { irradiated control group) }\end{array}$ & Vinagre et al. (2013) (28) & Melo et al. (2014) (25) \\
\hline $\begin{array}{l}\text { Follicular adenomas and } \\
\text { adenomatous nodules (FTA) }(n(\%))\end{array}$ & $6 / 29(20.7)$ & $0 / 33(0)$ & $0 / 60(0)$ & - \\
\hline Papillary carcinoma (PTC) $(n(\%))$ & $3 / 32(9.2)$ & - & $13 / 169(7.7)$ & $25 / 332(7.5) *$ \\
\hline $\begin{array}{l}\text { Well-differentiated carcinoma } \\
(\text { FTC + PTC + WDC-NOS) }(n(\%))\end{array}$ & $4 / 34(11.8)$ & - & $22 / 233(9.4)$ & $37 / 402(9.2) *$ \\
\hline
\end{tabular}

*The cases presented in Melo et al. (25) study include part of the cases published in Vinagre et al. (28). 
for the sporadic cases $(9.2-22.5 \%)(25,26,27,29)$. This observation fits with those previously obtained on $B R A F$, NRAS mutations and RET/PTC rearrangements (11), as the frequency of such genetic alterations in this irradiated, late-onset cohort was also similar to what is found in the sporadic context. At variance with this, in earlyonset radiation-associated thyroid cancers, such as the Chernobyl cohorts, no TERTp mutations were detected in the carcinomas analysed $(25,30)$. The biggest series, with 51 cases, is from Liu (30) who concludes that this genetic event is not related to radiation in thyroid cancer. In the present study, besides exploring the effect of a different type of radiation (X-rays), we evaluated a series of lateonset carcinomas, as the latency period between exposure and diagnosis was more than 40 years.

Regarding the adenomas, we found that TERTp mutations were a frequent event (20.7\% of the patients) in the X-ray-exposed setting. Previous studies have shown a $0-2 \%$ prevalence in the non-irradiated context $(25,28,31$, $32)$; the $2 \%$ prevalence ( $1 / 58$ cases) has been reported by Wang et al. (31) who considered the mutation as an early genetic event in thyroid follicular tumour malignancy. In the present study, we confirmed the absence of TERTp mutations in non-exposed follicular adenomas by screening a new series of 33 sporadic adenomas. In the Chernobyl radiation context, similar to what was reported for carcinomas, the mutation was not found in adenomas also (25), in accordance with our previous results $(25,28)$. Therefore, at the best of our knowledge, this is the first report of TERTp mutations in thyroid tumours (either carcinomas or adenomas) associated with X-ray external radiation exposure.

Furthermore, in our ten mutated cases, it is interesting to emphasise that $30 \%$ of the mutations were tandem mutations. This was confirmed by the absence of TERTp polymorphisms or mutations in the patient's blood. In thyroid tissues, these mutations have been reported only in BCPAP cell line $(26,28)$ and are infrequent in other cancer models $(26,33,34,35)$. Interestingly, in basal cell carcinomas from the patients of this tinea capitis cohort, we have found a different pattern, with a lower frequency of the $-124 \mathrm{G}>\mathrm{A}$ and a higher frequency of the $-146 \mathrm{G}>\mathrm{A}$ mutation, comparing with the sporadic BCC cases (34). In the present study, all the mutated cases were in the same $-124 /-125$ hotspot; no cases presented the $-146 \mathrm{G}>\mathrm{A}$ mutation. This is also in agreement with what has been described by others in sporadic thyroid cancer, as the $-146 \mathrm{G}>\mathrm{A}$ is the less frequent one $(10-17.2 \%)(25,27)$ or absent (26). This difference may be due to a different field effect, namely due to differences in the organs involved (skin vs thyroid) and differences in the dose received (5-6 Gy on the skin - an intermediate dose - compared to $0.5 \mathrm{~Gy}$, at maximum, on the thyroid - a low dose).

Comparing the clinicopathological characteristics of the mutated and non-mutated tumours (adenomas and carcinomas), we did not find any significant differences regarding age at irradiation, age at diagnosis and latency period. These negative results should be interpreted with caution due to the restricted number of mutated cases.

TERTp mutations were found to be frequent in adenomas from X-ray-irradiated patients, comparing to the $0-2 \%$ detected in the sporadic context $(28,31)$ and to the $0 \%$ in the Chernobyl setting (25), and showing a frequency similar to that one found in carcinomas. These results raise the possibility that the TERTp mutation may be an early genetic event in this context as advanced by Wang et al. (31). Curiously, such event does not appear to drive carcinogenesis, at least per se, as TERTp mutations are not more prevalent in the carcinomas arising in this setting than in sporadically occurring well-differentiated (papillary and follicular) carcinomas. This apparent dissociation between TERTp mutation and carcinogenesis in the X-irradiation context is reinforced by the finding of two cases in which TERTp mutation was detected in adenomas of the patients and not in the concurrent PTCs. Moreover, this also raises a very interesting point, which is the need to find the partner or partners that, together with TERTp mutation, may induce tumourigenesis.

The data herein presented suggest that TERTp mutations may represent a genetic signature for thyroid tumours submitted to low-dose X-ray external radiation as they were detected both in adenomas and in different histotypes of carcinoma. Moreover, in 30\% of the mutated cases, we observed the tandem mutation $-124 /-125 \mathrm{GG}>\mathrm{AA}$, which is to the best of our knowledge, never described before in thyroid tumours. It will be interesting to explore other thyroid low-dose radiation settings to see if the tandem mutation $-124 /-125 G G>A A$ is specific for this radiation model.

\section{Declaration of interest}

The authors declare that there is no conflict of interest that could be perceived as prejudicing the impartiality of the research reported.

\section{Funding}

This work was supported with funding from Prize ACS-MERCK SERONO in Cancer Epidemiology, 2010. Funding to P B was obtained from FCT/MEC through National Funds and co-financed by the FEDER through the PT2020 Partnership Agreement under the project $n^{\circ} 007274$ (UID/BIM/04293). This work was financed by FEDER - Fundo Europeu de Desenvolvimento Regional funds through the COMPETE 2020 - Operacional Programme for 
Competitiveness and Internationalisation (POCI), Portugal 2020 and by Portuguese funds through FCT - Fundação para a Ciência e a Tecnologia/ Ministério da Ciência, Tecnologia e Inovação in the framework of the project 'Institute for Research and Innovation in Health Sciences' (POCl01-0145-FEDER-007274). Further funding from the project 'Advancing cancer research: from basic knowledgment to application'; NORTE-010145-FEDER-000029; 'Projetos Estruturados de I\&D\&I', funded by Norte 2020 - Programa Operacional Regional do Norte; IPATIMUP integrates the i3S Research Unit, which is partially supported by FCT, the Portuguese Foundation for Science and Technology. This work is funded by FEDER funds through the Operational Programme for Competitiveness Factors COMPETE and National Funds through the FCT, under the projects 'PEst-C/ SAU/LA0003/2013'. The funding sources had no involvement in study design, collection, analysis and interpretation of data; in the writing of the report and in the decision to submit the article for publication.

\section{Acknowledgements}

The authors are grateful to all patients who agreed to participate in this study as well as to all the physicians who provided clinical information.

\section{References}

1 Boaventura P, Pereira D, Mendes A, Teixeira-Gomes J, SobrinhoSimões M \& Soares P. Thyroid and parathyroid tumours in patients submitted to X-ray scalp epilation during the tinea capitis eradication campaign in the North of Portugal (1950-1963). Virchows Archiv: European Journal of Pathology 2014465 445-452. (doi:10.1007/s00428014-1644-0)

2 Crossland PM. Therapy of tinea capitis; the value of x-ray epilation. California Medicine 195684 351-353.

3 Brandão N. A roentgenterapia das tinhas do couro cabeludo (Aspectos técnicos e problemas). O Médico 19531 857-864.

4 Ron E, Modan B, Preston D, Alfandary E, Stovall M \& Boice JD Jr. Radiation-induced skin carcinomas of the head and neck. Radiation Research 1991125 318-325. (doi:10.2307/3578117)

5 Sadetzki S, Chetrit A, Freedman L, Stovall M, Modan B \& Novikov I. Long-term follow-up for brain tumor development after childhood exposure to ionizing radiation for tinea capitis. Radiation Research 2005163 424-432. (doi:10.1667/RR3329)

6 Sadetzki S, Chetrit A, Lubina A, Stovall M \& Novikov I. Risk of thyroid cancer after childhood exposure to ionizing radiation for tinea capitis. Journal of Clinical Endocrinology and Metabolism 200691 4798-4804. (doi:10.1210/jc.2006-0743)

7 Suzuki K, Mitsutake N, Saenko V \& Yamashita S. Radiation signatures in childhood thyroid cancers after the Chernobyl accident: possible roles of radiation in carcinogenesis. Cancer Science 2014106 127-133. (doi:10.1111/cas.12583)

8 Boaventura P, Oliveira R, Pereira D, Soares P \& Teixeira-Gomes J. Head and neck basal cell carcinoma prevalence in individuals submitted to childhood X-ray epilation for tinea capitis treatment. European Journal of Dermatology 201222 225-230.

9 Shore RE, Moseson M, Xue X, Tse Y, Harley N \& Pasternack BS. Skin cancer after X-ray treatment for scalp ringworm. Radiation Research 2002157 410-418. (doi:10.1667/0033-7587(2002)157[0410:SCAXRT] 2.0.CO;2)

10 Boaventura P, Soares P, Pereira D, Teixeira-Gomes J \& SobrinhoSimões M. Head and neck lesions in a cohort irradiated in childhood for tinea capitis treatment. Lancet Infectious Diseases 201111 163-164. (doi:10.1016/S1473-3099(11)70047-0)

11 Boaventura P, Pereira D, Celestino R, Mendes A, Nakasawa T, TeixeiraGomes J, Sobrinho-Simões M \& Soares P. Genetic alterations in thyroid tumors from patients irradiated in childhood for tinea capitis treatment. European Journal of Endocrinology $2013169673-679$ (doi:10.1530/EJE-13-0543)

12 Nikiforova MN, Ciampi R, Salvatore G, Santoro M, Gandhi M, Knauf JA, Thomas GA, Jeremiah S, Bogdanova TI, Tronko MD et al. Low prevalence of BRAF mutations in radiation-induced thyroid tumors in contrast to sporadic papillary carcinomas. Cancer Letters 2004209 1-6. (doi:10.1016/j.canlet.2003.12.004)

13 Lima J, Trovisco V, Soares P, Máximo V, Magalhães J, Salvatore G, Santoro M, Bogdanova T, Tronko M, Abrosimov A et al. BRAF mutations are not a major event in post-Chernobyl childhood thyroid carcinomas. Journal of Clinical Endocrinology and Metabolism 200489 4267-4271. (doi:10.1210/jc.2003-032224)

14 Fugazzola L, Puxeddu E, Avenia N, Romei C, Cirello V, Cavaliere A, Faviana P, Mannavola D, Moretti S, Rossi S et al. Correlation between B-RAFV600E mutation and clinico-pathologic parameters in papillary thyroid carcinoma: data from a multicentric Italian study and review of the literature. Endocrine-Related Cancer 200613 455-464. (doi:10.1677/erc.1.01086)

15 Nikiforov YE, Rowland JM, Bove KE, Monforte-Munoz H \& Fagin JA. Distinct pattern of ret oncogene rearrangements in morphological variants of radiation-induced and sporadic thyroid papillary carcinomas in children. Cancer Research 199757 1690-1694.

16 Williams GH, Rooney S, Thomas GA, Cummins G \& Williams ED. RET activation in adult and childhood papillary thyroid carcinoma using a reverse transcriptase-n-polymerase chain reaction approach on archival-nested material. British Journal of Cancer $1996 \mathbf{7 4}$ 585-589. (doi:10.1038/bjc.1996.405)

17 Elisei R, Romei C, Vorontsova T, Cosci B, Veremeychik V, Kuchinskaya E, Basolo F, Demidchik EP, Miccoli P, Pinchera A et al. RET/PTC rearrangements in thyroid nodules: studies in irradiated and not irradiated, malignant and benign thyroid lesions in children and adults. Journal of Clinical Endocrinology and Metabolism 200186 3211-3216.

18 Tronko M, Bogdanova T, Voskoboynyk L, Zurnadzhy L, Shpak V \& Gulak L. Radiation induced thyroid cancer: fundamental and applied aspects. Experimental Oncology 201032 200-204.

19 Williams D. Twenty years' experience with post-Chernobyl thyroid cancer. Best Practice and Research Clinical Endocrinology and Metabolism 200822 1061-1073. (doi:10.1016/j.beem.2008.09.020)

20 Williams ED, Abrosimov A, Bogdanova T, Demidchik EP, Ito M, LiVolsi V, Lushnikov E, Rosai J, Sidorov Y, Tronko MD et al. Thyroid carcinoma after Chernobyl latent period, morphology and aggressiveness. British Journal of Cancer 200490 2219-2224.

21 Dinets A, Hulchiy M, Sofiadis A, Ghaderi M, Hoog A, Larsson C \& Zedenius J. Clinical, genetic, and immunohistochemical characterization of 70 Ukrainian adult cases with post-Chornobyl papillary thyroid carcinoma. European Journal of Endocrinology 2012 166 1049-1060. (doi:10.1530/EJE-12-0144)

22 Hanahan D \& Weinberg RA. Hallmarks of cancer: the next generation. Cell 2011144 646-674. (doi:10.1016/j.cell.2011.02.013)

23 Vinagre J, Pinto V, Celestino R, Reis M, Pópulo H, Boaventura P, Melo M, Catarino T, Lima J, Lopes JM et al. Telomerase promoter mutations in cancer: an emerging molecular biomarker? Virchows Archiv: European Journal of Pathology 2014465 119-133. (doi:10.1007/ s00428-014-1608-4)

24 Ngeow J \& Eng C. TERT and BRAF in thyroid cancer: teaming up for trouble. Journal of Clinical Oncology 201432 2683-2684. (doi:10.1200/ JCO.2014.56.5614)

25 Melo M, da Rocha AG, Vinagre J, Batista R, Peixoto J, Tavares C, Celestino R, Almeida A, Salgado C, Eloy C et al. TERT promoter mutations are a major indicator of poor outcome in differentiated thyroid carcinomas. Journal of Clinical Endocrinology and Metabolism 201499 E754-E765. (doi:10.1210/jc.2013-3734)

26 Liu X, Bishop J, Shan Y, Pai S, Liu D, Murugan AK, Sun H, El-Naggar AK \& Xing M. Highly prevalent TERT promoter mutations 
in aggressive thyroid cancers. Endocrine-Related Cancer 201320 603-610. (doi:10.1530/ERC-13-0210)

27 Landa I, Ganly I, Chan TA, Mitsutake N, Matsuse M, Ibrahimpasic T, Ghossein RA \& Fagin JA. Frequent somatic TERT promoter mutations in thyroid cancer: higher prevalence in advanced forms of the disease. Journal of Clinical Endocrinology and Metabolism 201398 E1562-E1566. (doi:10.1210/jc.2013-2383)

28 Vinagre J, Almeida A, Pópulo H, Batista R, Lyra J, Pinto V, Coelho R, Celestino R, Prazeres H, Lima L et al. Frequency of TERT promoter mutations in human cancers. Nature Communications 201342185. (doi:10.1038/ncomms3185)

29 Muzza M, Colombo C, Rossi S, Tosi D, Cirello V, Perrino M, De Leo S, Magnani E, Pignatti E, Vigo B et al. Telomerase in differentiated thyroid cancer: promoter mutations, expression and localization. Molecular and Cellular Endocrinology 2015399 288-295. (doi:10.1016/j. mce.2014.10.019)

30 Liu T. Telomerase Reverse Transcriptase (TERT): Promoter Mutation and Novel Function in Human Cancer, p 72. Stockholm, Sweden: Karolinska University Hospital Solna, 2014.

31 Wang N, Liu T, Sofiadis A, Juhlin CC, Zedenius J, Hoog A, Larsson C $\& \mathrm{Xu}$ D. TERT promoter mutation as an early genetic event activating telomerase in follicular thyroid adenoma (FTA) and atypical FTA. Cancer 2014120 2965-2979. (doi:10.1002/cncr.28800)

32 Liu X, Qu S, Liu R, Sheng C, Shi X, Zhu G, Murugan AK, Guan $\mathrm{H}, \mathrm{Yu} \mathrm{H}$, Wang $\mathrm{Y}$ et al. TERT promoter mutations and their association with BRAF V600E mutation and aggressive clinicopathological characteristics of thyroid cancer. Journal of Clinical Endocrinology and Metabolism 201499 E1130-E1136. (doi:10.1210/jc.2013-4048)

33 Heidenreich B, Nagore E, Rachakonda PS, Garcia-Casado Z, Requena C, Traves V, Becker J, Soufir N, Hemminki K \& Kumar R. Telomerase reverse transcriptase promoter mutations in primary cutaneous melanoma. Nature Communications 201453401. (doi:10.1038/ncomms4401)

34 Pópulo H, Boaventura P, Vinagre J, Batista R, Mendes A, Caldas R, Pardal J, Azevedo F, Honavar M, Guimarães I et al. TERT promoter mutations in skin cancer: the effects of sun exposure and $\mathrm{X}$-irradiation. Journal of Investigative Dermatology $2014 \mathbf{1 3 4}$ 2251-2257. (doi:10.1038/jid.2014.163)

35 Liu T, Yuan X \& Xu D. Cancer-specific telomerase reverse transcriptase (TERT) promoter mutations: biological and clinical implications. Genes 20167 1-18.

Received 30 August 2016

Revised version received 15 October 2016

Accepted 19 October 2016 